\title{
STRUCTURE OF FOURIER AND FOURIER-STIELTJES COEFFICIENTS OF SERIES WITH SLOWLY VARYING CONVERGENCE MODULI
}

\author{
ČASLAV V. STANOJEVIĆ
}

Ostensibly, convergence problems regarding the Fourier series in $L^{\mathbf{1}}$ parallel the classical Tauberian problems. Let $f \in L^{1}(T), T=\mathbf{R} / 2 \pi \mathbf{Z}$, then the partial sums $S_{n}(f)=S_{n}(f, t)=\sum_{|k| \leq n} \hat{f}(k) e^{i k t}$ are $(C, 1)$-summable, both pointwise and in $L^{1}$-norm. Inasmuch as the appropriate Tauberian conditions are available, the convergence questions may be settled in the standard manner. However, Tauberian conditions needed to recover $L^{1}$-convergence originate from the Hausdorff-Young inequality and do not have a straightforward analogue in the elementary Tauberian theory. Such a condition is obtained in [1], i.e.

$$
\lim _{\lambda \rightarrow 1+0} \varlimsup_{n} \sum_{|k|=n+1}^{[\lambda n]}|k|^{p-1}|\Delta \widehat{f}(k)|^{p}=0,
$$

where $1<p \leq 2$ and $f \in L^{1}(T)$. Later in [2 and 3], the condition (1) has been further extended and studied. Although (1) is much weaker than the classical $[4,5]$ and neoclassical $[6,7]$ regularity and/or speed conditions, it does not provide explicit information about the Fourier coefficients. To overcome this shortcoming a new approach is proposed in [8], based on regular variation of the convergence moduli. if

A nondecreasing sequence $\{R(n)\}$ of positive numbers is *-regularly varying

$$
\lim _{\lambda \rightarrow 1+0} \varlimsup_{n} \frac{R([\lambda n])}{R(n)} \leq 1
$$

or more generally, the sequence $\{R(n)\}$ is $O$-regularly varying if

$$
\varlimsup_{n} \frac{R([\lambda n])}{R(n)}
$$

is finite for $\lambda>1$. In particular, if $\lim _{n} R([\lambda n]) / R(n)=1,\{R(n)\}$ is slowly varying.

Let $\{c(n)\}$ be a sequence of complex numbers and let $\sum_{|n|<\infty} c(n) e^{i n t}$ be its formal trigonometric transform. The convergence modulo of the trigonometric transform is defined as

$$
K_{n}^{p}(c)=\sum_{|k| \leq n}|k|^{p-1}|\Delta c(k)|^{p}, \quad p>1 .
$$

Received by the editors October 15, 1987.

1980 Mathematics Subject Classification (1985 Revision). Primary 42A20, 42A32. 
The following two theorems are proved in [8]. In the second theorem $M(T)$ denotes the class of all complex Borel measures on $T$.

THEOREM A. Let $f \in L^{1}(T)$ and for some $1<p \leq 2$ and some *-regularly varying sequence $\{R(n)\}$ let $K_{n}^{p}(f)=\log R(n)$. Then the Fourier series of $f$ converges a.e. and it converges in $L^{1}(T)$-norm if and only if $\widehat{f}(n) \log |n|=$ $o(1),|n| \rightarrow \infty$.

THEOREM B. Let $\mu \in M(T)$ and let $(\widehat{\mu}(n)-\widehat{\mu}(-n)) \log n=O(1), n \rightarrow$ $\infty$. If for some $1<p \leq 2$ and some $O$-regularly varying sequence $\{R(n)\}$, $K_{n}^{p}(\mu)=\log R(n)$, then

$$
\left\|S_{n}(\mu)\right\|=B_{n}|\widehat{\mu}(n)| \log n+O(1), \quad n \rightarrow \infty,
$$

where $\left\{B_{n}\right\}$ is a bounded sequence of real numbers, bounded away from zero.

This theorem is a quantitative version of Helson's [9] theorem, and it is given here in somewhat different form than in [8] to match the form of Theorem A.

In this announcement we shall show that: (i) *-regular variation in Theorem A can be lightened to $O$-regular variation; and (ii) there is a representation for $\{\widehat{f}(n)\}$ and $\{\widehat{\mu}(n)\}$ in terms of Fourier coefficients of functions in $L^{q}(T), 1 / p+1 / q=1$. Consequently, Theorem A and Theorem B can be unified in a new theorem that improves both theorems, in particular Theorem A.

THEOREM. Let $\{c(n)\}_{|n|<\infty}$ be a sequence of complex numbers and for some $1<p \leq 2$ and some $O$-regularly varying sequence $\{R(n)\}$ let

$$
K_{n}^{p}(c)=\log R(n) .
$$

Then

(i) there exists $h \in L^{q}(T), 1 / p+1 / q=1$, such that

$c(k)=\frac{1+\operatorname{sgn}(k)}{2} c(0)+\frac{1-\operatorname{sgn}(k)}{2} c(-1)-\sum_{j=(1-\operatorname{sgn}(k)) / 2}^{|k|-1} \widehat{h}(j \operatorname{sgn}(k)), \quad k \in \mathbf{Z}$;

(ii) for $c(n)=o(1),|n| \rightarrow \infty$, the trigonometric transform of $\{c(n)\}$ converges a.e. and $c(k)=\sum_{j=|k|}^{\infty} \widehat{h}(j \operatorname{sgn}(k)), k \in \mathbf{Z}$;

(iii) for $c=\widehat{f}, f \in L^{1}(T)$, the Fourier series of $f$ converges in $L^{1}(T)$-norm if and only if $\widehat{f}(n) \log |n|=o(1),|n| \rightarrow \infty$.

(iv) for $c=\widehat{\mu}, \mu \in M(T)$, and $(\widehat{\mu}(n)-\widehat{\mu}(-n)) \log n=O(1), n \rightarrow \infty$;

$$
\left\|S_{n}(\mu)\right\|=B_{n}|\widehat{\mu}(n)| \log n+O(1), \quad n \rightarrow \infty,
$$

where $\left\{B_{n}\right\}$ is a bounded sequence of real numbers, bounded away from zero.

OUTLINE OF PROOF. The case $K_{n}^{p}(c)=O(1), n \rightarrow \infty$, is trivial. Assume $K_{n}^{p}(c) \rightarrow \infty, n \rightarrow \infty$. Then condition (2) is equivalent to:

$$
\varlimsup_{n} \sum_{|k|=n+1}^{[\lambda n]}|k|^{p-1}|\Delta c(k)|^{p} \quad \text { is finite for } \lambda>1 \text {. }
$$


Hence $\left\{K_{n}^{p}(c)\right\}$ is slowly varying. Also

$$
\sum_{|j| \leq n}|\Delta c(j)|^{p} \leq C_{1} \sum_{|j| \leq n} \frac{K_{j}^{p}(c)}{|j|^{p}}+C_{2} \frac{K_{n}^{p}(c)}{|n|^{p-1}},
$$

where $C_{1}$ and $C_{2}$ are absolute constants. The series on the left-hand side of (3) is therefore convergent. By F. Riesz's theorem [10] there is an $h \in L^{q}(T)$, $1 / p+1 / q=1$, such that

$$
h(t)=\sum_{|n|<\infty} \Delta c(n) e^{i n t},
$$

and the representation in (i) follows.

The series (4) converges a.e. by L. Carleson's theorem [11], for $c(n)=o(1)$, $|n| \rightarrow \infty$, and for $t \neq 0, \sum_{|n|<\infty} c(n) e^{i n t}$ converges a.e. The representation in (ii) is now evident.

The statement (iii) is a considerable generalization of Theorem 2.1 in [8] and the proof requires several refinements. Let $\lambda>1$. Define

$$
T_{n}(\lambda)=(-\pi,-\pi /(\lambda-1) n) \cup(\pi /(\lambda-1) n, \pi)
$$

and

$$
\tau_{n}(f, \lambda)=\tau_{n}(f, t, \lambda)=\frac{1}{[\lambda n]-n} \sum_{k=n+1}^{[\lambda n]} S_{k}(f)
$$

Then

$$
\begin{aligned}
\left\|\tau_{n}(f, \lambda)-S_{n}(t)\right\|_{L^{1}(t)}= & \int_{T_{n}(g)}\left|\tau_{n}(f, t, \lambda)-S_{n}(f, t)\right| d t \\
& +\int_{T-T_{n}(\lambda)}\left|\tau_{n}(f, t, \lambda)-S_{n}(f, t)\right| d t \\
= & J_{1}+J_{2} .
\end{aligned}
$$

For $J_{2}$ we have the uniform estimates

$$
J_{2} \leq \frac{1}{[\lambda n]-n} \sum_{|k|=n+1}^{[\lambda n]}|\widehat{f}(k)|=o(1), \quad n \rightarrow \infty .
$$

The integral $J_{1}$ can be written as a sum of two integrals $J_{11}$ and $J_{12}$. Applying Hölder's inequality to $J_{11}$, followed by the Hausdorff-Young inequality, we get

$$
J_{11} \leq C_{3}(\lambda-1)^{1 / q} \sum_{|k|=n+1}^{[\lambda n]}|k|^{p-1}|\Delta \widehat{f}(k)|^{p},
$$

where $C_{3}$ is an absolute constant. Thus

$$
\lim _{\lambda \rightarrow 1+0} \varlimsup_{n} J_{11}=0 \text {. }
$$

Therefore

$$
\lim _{\lambda \rightarrow 1+0} \varlimsup_{n}\left\|\tau_{n}(f, \lambda)-S_{n}(f)\right\|_{L^{1}(T)}=0
$$


is equivalent to

$$
\lim _{\lambda \rightarrow 1+0} \varlimsup_{n} J_{12}=0 .
$$

However

$$
J_{12}=\int_{T_{n}(\lambda)}\left|\frac{\widehat{f}(n) e^{i n t}+\widehat{f}(-n) e^{-i n t}}{1-e^{-i t}}\right| d t,
$$

and $\varlimsup_{n} J_{12}=0$ if and only if $\widehat{f}(n) \log |n|=o(1),|n| \rightarrow \infty$. Hence (4) is equivalent to $\widehat{f}(n) \log |n|=o(1),|n| \rightarrow \infty$. A standard argument completes the proof.

The proof of (iv) follows the lines of the proof of Theorem 2.3 in [8].

The details and proofs will appear elsewhere.

\section{REFERENCES}

1. C. V. Stanojevic, Tauberian conditions for the $L^{1}$-convergence of Fourier series, Trans. Amer. Math. Soc. 271 (1982), 237-244.

2. W. O. Bray and C. V. Stanojević, Tauberian $L^{1}$-convergence classes of Fourier series. I, Trans. Amer. Math. Soc. 275 (1983), 59-69.

3. __ Tauberian $L^{1}$-convergence classes of Fourier series. II, Math. Ann. 269 (1984), 469-486.

4. S. Sidon, Hinreichende Bedingungen fur den Fourier Charakter einer Trigonometrischen Reihe, J. London Math. Soc. (2) 14 (1939), 158-160.

5. S. A. Telyakovskii, On a sufficient condition of Sidon for the integrability of trigonometric series, Math. Notes 14 (1973), 742-748.

6. G. A. Fomin, A class of trigonometric series, Math. Notes 23 (1978), 117-124.

7. C. V. Stanojevic and V. B. Stanojevic, Generalizations of the Sidon-Telyakovskii Theorem, Proc. Amer. Math. Soc. 101 (1987), 679-684.

8. ¿. V. Stanojevic, $O$-Regularly varying convergence moduli of Fourier and Fourier-Stieltjes series, Math. Ann. 279 (1987), 103-115.

9. H. Helson, Proof of a conjecture of Steinhaus, Proc. Nat. Acad. Sci. U.S.A. 40 (1954), 205-206.

10. F. Riesz, Über eine Verallgemeinerung des Parsevalschen Formel, Math. Z. 18 (1923), 117-124.

11. L. Carleson, On convergence and growth of partial sums of Fourier series, Acta Math. 116 (1966), 135-157.

UNIVERSITY OF MISSOURI-ROLLA, ROLLA, MISSOURI 65401 